\title{
On the Relationship between the Geometry of Space-Time and Its Information Content
}

\author{
Andreas E. Schlatter \\ Burghaldeweg 2F, Küttigen, Switzerland \\ Email: schlatter.a@bluewin.ch \\ Received 3 November 2015; accepted 6 December 2015; published 9 December 2015 \\ Copyright (C) 2015 by author and Scientific Research Publishing Inc. \\ This work is licensed under the Creative Commons Attribution International License (CC BY). \\ http://creativecommons.org/licenses/by/4.0/ \\ (c) (i) Open Access
}

\begin{abstract}
We use an information-consistency or, equivalently, a thermodynamic equilibrium condition to derive Einstein's equations, both in case of a gravitational and an electrostatic field. We thus show the equivalence of an information-theoretic and a thermodynamic viewpoint in the analysis of the geometry of space-time.
\end{abstract}

\section{Keywords}

\section{Entropy, Quantum Gravity, Information, Einstein Equations}

\section{Introduction}

In recent years, we have seen the demonstration of profound relationships between the geometry of space-time and thermodynamics or quantum information [1] [2]. In all cases it results in a derivation of Einstein's equations as a consequence either of a thermodynamic equilibrium condition at a causal horizon, or of counting the number of possible flips in a space-time volume and changing the area of a space-like surface by a suitable multiple of that number. In this paper we are going to combine these approaches to show that Einstein's equations actually follow from an information consistency condition under a thermal evolution. In addition we will also see that it does not make a difference whether we consider a mass and its gravitational field or a charge and its Coulomb and (induced) magnetic fields. Both sources of energy influence the geometry of space-time by the same mechanism.

There has been recent work done around the concept of thermal time and its relations to other, geometric time-flows [3]-[7]. We will make the thermal flow of a physical system, represented by a density operator $\rho_{0}$, the starting point of our considerations. 


\section{Process Velocity}

Let there be a physical system with density operator $\rho_{0}=\varrho(0)$ and a Hamiltonian $H$. The cosine of the angle between the initial state $\varrho_{0}$ and any further state $\varrho(t)=\mathrm{e}^{-\frac{i}{\hbar} H t} \rho_{0} \mathrm{e}^{\frac{i}{\hbar} H t}, t>0$, under the Schroedinger evolution is given by the scalar product $\left\langle\rho_{0}, \rho(t)\right\rangle$. A future state $\rho(\tau)$ is called distinguishable from $\varrho_{0}$, if it is orthogonal, i.e. $\left\langle\varrho_{0}, \varrho(\tau)\right\rangle=0$. The minimal time $\tau$ needed for $\varrho_{0}$ to evolve into an orthogonal state is given by the Margolus-Levitin bound [8],

$$
\tau=\frac{h}{4 \bar{E}} .
$$

Here $\bar{E}$ denotes the average energy $\bar{E}=\operatorname{tr}\left(\rho_{0} H\right)$ of the system ${ }^{1}$ and $h$ denotes the Planck constant. If, for instance, the number of degrees of freedom of the system is large, we may assume that the value $2 \bar{E}$ is part of the spectrum and hence the minimum-time $\tau$ is actually attained. Since general relativity is a macroscopic theory, this will most likely hold for a system $\rho_{0}$. If, instead of flipping between two orthogonal states, one considers passing through a long sequence of orthogonal states, then the minimal time turns out to be $\tau=\frac{h}{2 \bar{E}}$.

We will make use of both results.

We now suppose that the system is part of a heat bath $\Omega, \varrho_{0} \subset \Omega$, at temperature $T$ and consider the flow generated under the assumption that the density operator $\varrho_{0}$ represents itself thermal equilibrium, i.e. maximum entropy, and the Hamiltonian is therefore $H=-k T \log _{2} \varrho_{0}$, where $k$ denotes the Boltzmann constant. By using this Hamiltonian Equation (1) turns into

$$
\tau=-\frac{h}{4 k T \cdot E_{\rho_{0}}\left(\log _{2} \rho_{0}\right)}=-\frac{h}{4 k T \cdot \operatorname{tr}\left(\rho_{0} \log _{2} \rho_{0}\right)}=\frac{h}{4 k T S_{0}} .
$$

In this equation $S_{0}$ denotes the (von Neumann) entropy of the system. Equation (2) allows us to define a (process) velocity, i.e. the average number of orthogonal states passed by unit of (proper) time $\mathrm{ds}$

$$
\frac{\mathrm{d} N_{\varrho_{0}}}{\mathrm{~d} s}=\frac{1}{\tau}=\frac{4 k T S_{\varrho_{0}}}{h} .^{2}
$$

Expression (3) further allows us to define a very general equilibrium condition between two systems [6]. Given two systems $\varrho$ and $\varrho$ with their respective temperatures $T_{\varrho}$ and $T_{\hat{\varrho}}$, and corresponding observers with their proper-time intervals $\mathrm{d} s_{\varrho}$ and $\mathrm{d} s_{\hat{\varrho}}$, we say that two systems are in equilibrium relative to these observers, if there holds

$$
\mathrm{d} N_{\varrho}=\mathrm{d} N_{\hat{\varrho}} \leftrightarrow T_{\rho} S_{\varrho} \mathrm{d} s_{\varrho}=T_{\hat{\varrho}} S_{\hat{\varrho}} \mathrm{d} s_{\hat{\varrho}} .
$$

Note that condition (4) can also be viewed as a consistency condition for two sub-systems of an overall system in thermal equilibrium, as indicated in [9]. It is important that with a relation like (4) we actually consider classical information in form of distinguishable states, which appears natural, if a macroscopic theory like relativity is in scope.

\section{Information and Space-Time}

Equation (4) is a relational expression, which we now apply to the situation of observers in a four-dimensional space-time. A space-time allows an equilibrium, if it is static [6] [10], i.e. if its local line-element does not explicitly depend upon the time-coordinate and is therefore of the form

\footnotetext{
${ }^{1}$ Expression (1) assumes that the lowest eigenvalue $E_{0}=0$, otherwise there holds $\tau=\frac{h}{4\left(\bar{E}-E_{0}\right)}$.

${ }^{2}$ Note that at this place the definition of temperature as the average energy per bit of information, $T=\frac{\partial E}{\partial S}$, appears very natural.
} 


$$
\mathrm{d} s^{2}=g_{00}(x) \mathrm{d} t^{2}+\sum_{i, j} g_{i j}(x) \mathrm{d} x_{i} \mathrm{~d} x_{j} .
$$

From Equation (5) it is immediately evident that for two observers along their time-like world-lines with $\mathrm{d} s_{1 / 2}=\sqrt{g_{o o}\left(x_{1 / 2}\right)} \mathrm{d} t$ and $S_{\varrho}=S_{\hat{\varrho}}$, relation (4) turns into

$$
T\left(x_{1}\right) \sqrt{g_{00}\left(x_{1}\right)}=T\left(x_{2}\right) \sqrt{g_{00}\left(x_{2}\right)} .
$$

This is the well-known Tolman-Ehrenfest effect [10]. This elegant derivation already indicates the usefulness of definition (4).

We now consider a situation, where the space-time is actually a flat Minkowski space-time and the observers move with constant acceleration. To generate a static environment we work in the Rindler co-moving coordinate frame and hence in the Rindler-wedge. In Rindler-coordinates $(\theta, \lambda, y, z)$ the line-element takes the form

$$
\mathrm{d} s^{2}=c^{2} \mathrm{e}^{2 \lambda} \mathrm{d} \theta^{2}-\mathrm{e}^{2 \lambda} \mathrm{d} \lambda^{2}-\mathrm{d} y^{2}-\mathrm{dz} z^{2} .
$$

For the constant acceleration $a$ there holds $\mathrm{e}^{\lambda}=\frac{1}{a} \cdot{ }^{3}$

For an observer along a time-like world-line with $\mathrm{d} s=\frac{c}{a} \mathrm{~d} \theta$ and a system with $S_{\varrho}=1$ relations (3) and (4) result in

$$
\frac{4 k}{h} T_{a} \cdot \frac{c}{a}=\text { const. }
$$

By a calculation to normalize states, the value chosen for the constant is const. $=\frac{1}{\pi^{2}}$ and Equation (8) turns into the Unruh-relation with corresponding Unruh temperature $T_{a} \cdot{ }^{4}$

\subsection{Gravity}

Our next step is now to consider a test particle of mass $m$ at a distance $R$ from a central object of mass $M$ at a point $p_{0}$ in space-time. Working at first in a flat space-time means that we can use the Newtonian form (approximation) of the gravity law to see that, with $G$ denoting the gravitational constant, the particle feels an acceleration of

$$
a:=g_{R}=\frac{G M}{R^{2}} .
$$

Along a small segment $\mathrm{d} \theta$ of the particle's world-line the acceleration can be thought to be constant. If we consider long sequences of orthogonal states to be passed, then relation (8) turns into the following chain of equalities

$$
\frac{2 k}{h} T_{g_{R}} \cdot \frac{c}{g_{R}}=\frac{2 k}{h} T_{g_{R}} \cdot \frac{c R^{2}}{G M}=\frac{2 k}{h} T_{g_{R}} \cdot \frac{A_{R}}{4 \pi} \cdot \frac{c}{G M}=\frac{1}{\pi^{2}} .
$$

Together with the definition of the Planck-length $l_{P}=\sqrt{\frac{G \hbar}{c^{3}}}$, (10) turns into

$$
k T_{g_{R}} \frac{A_{R}}{4 l_{P}^{2}}=M c^{2} .
$$

An expression, which originally served the purpose to count orthogonal states in equilibrium, thus turns into an energy-entropy relationship of the form $Q=k T S$, leaving us to formally identify entropy with the (horizon) surface

${ }^{3}$ For more information on Rindler-coordinates see e.g. [11].

${ }^{4}$ Since the equilibrium condition (4) is relational, there is always a gauge freedom of multiplication by a constant, non-zero function. 


$$
S=\frac{k A_{R}}{4 l_{P}^{2}}
$$

If we again use (8) to express temperature $T_{g_{R}}=\frac{\hbar}{2 \pi c k} \cdot g_{R}$, we finally get with total energy $E=M c^{2}$

$$
\frac{\hbar g_{R}}{c} \cdot \frac{A_{R}}{8 \pi l_{P}^{2}}=M c^{2}=E
$$

Note that, if energy is quantized, $E=n \cdot E_{0}, n \in \mathbb{N}$, then the same holds for the horizon $A_{R}=n \cdot \frac{E_{0}}{\gamma}$ with $\gamma=\frac{8 \pi l_{P}^{2}}{\hbar g_{R}}$.

In a general Lorentz space-time relation (13) is still locally valid, since we can in a small neighborhood around $p_{0}$ work in a Minkowski-frame. We now want to investigate what happens, if there is a change of mass (energy) $\delta M$ over the local causal horizon $\mathcal{H}$ at $p_{0}$, and the equilibrium is maintained, i.e.

$$
\frac{\hbar g_{R}}{8 \pi l_{P}^{2} C} \delta A_{R}=\delta M c^{2}=\delta E .
$$

Let us shortly summarize before we go on. Our formula to count the (maximal) number of orthogonal states (bits) per unit-time under a thermal evolution (3), together with a consistency or equilibrium definition across different observers (4), applied to a test particle in a gravitational field in the Newtonian approximation (inverse-square law), has lead to Equation (13) and Equation (14) which allows us to formally identify entropy with a surface area and establish an equilibrium relation with total energy. This is quite remarkable and delivers the preconditions to follow the approach in e.g. [1] [12]. We start with rewriting Equation (14)

$$
g_{R} \cdot \delta A_{R}=\frac{8 \pi l_{P}^{2} C}{\hbar} \cdot \delta E=\tilde{\alpha} \cdot \delta E,
$$

and for completeness sake sketch the steps.

We may chose $R$ in such a way, ${ }^{5}$ that in a small neighborhood of any space-like 2-surface $P$ around the space-time point $p_{0} \in P$, space-time is approximately flat and there is an approximate local boost Killing-field $\chi^{v}$, future-pointing to the inside past of $p_{0}$ and with acceleration $g_{R}$, generating a horizon $\mathcal{H}$. If $k^{v}$ is the tangent vector to the horizon generators, then for an affine parameter $\lambda$ that vanishes at $P$, there holds for $\lambda$ small enough $\chi^{v}=-g_{R} \lambda k^{v}$. We therefore get for the horizon volume-element $\mathrm{d} \mathcal{H}^{v}=k^{v} \mathrm{~d} \lambda \mathrm{d} A$ and for the energy flow through the horizon together with the (matter)energy tensor $T_{\mu v}{ }^{6}$

$$
\delta E=-g_{R} \int \lambda T_{\mu \nu} k^{\mu} k^{v} \mathrm{~d} \lambda \mathrm{d} A .
$$

For the variation of the horizon area there holds with $\theta$ denoting the expansion of the horizon generators

$$
\delta A=\int \theta \mathrm{d} \lambda \mathrm{d} A .
$$

Now, the equation of geodesic deviation, applied to the null geodesic congruence generating the horizon, leads to the Raychaudhuri equation [13].

$$
\frac{\mathrm{d} \theta}{\mathrm{d} \lambda}=-\frac{1}{2} \theta^{2}-\sigma^{2}-R_{\mu \nu} k^{\mu} k^{\nu}
$$

where $\sigma$ denotes the shear and $R_{\mu v}$ the Ricci-tensor. Both, $\theta$ and $\sigma$ disappear, since we have chosen our local Rindler-horizon to be instantaneously stationary at $P$, and therefore for $\lambda$ sufficiently small there holds $\theta \approx-\lambda R_{\mu \nu} k^{\mu} k^{v}$. Putting this into Equation (17) we get with sufficient accuracy

$$
\delta A=-\int \lambda R_{\mu \nu} k^{\mu} k^{v} \mathrm{~d} \lambda \mathrm{d} A .
$$

${ }^{5}$ Typically $R$ will be small and hence the accelereation $g_{R}$ big.

${ }^{6}$ We assume that all energy is transported through the horizon by a matter flux. 
Now, by Equation (15) there holds $g_{R} \cdot \delta A=\tilde{\alpha} \cdot \delta E$ and hence

$$
-g_{R} \int \lambda R_{\mu \nu} k^{\mu} k^{v} \mathrm{~d} \lambda \mathrm{d} A=-\tilde{\alpha} g_{R} \int \lambda T_{\mu \nu} k^{\mu} k^{v} \mathrm{~d} \lambda \mathrm{d} A .
$$

Therefore

$$
R_{\mu \nu} k^{\mu} k^{v}=\tilde{\alpha} T_{\mu \nu} k^{\mu} k^{v} .
$$

This holds for all null $k^{\mu}$, which implies that for some function $f$

$$
R_{\mu v}+f g_{\mu v}=\tilde{\alpha} T_{\mu v}
$$

Since $T_{\mu v}$ is divergence free, there holds by the contracted Bianchi identity $f=-\frac{R}{2}+\Lambda$, where $R$ denotes the Ricci-scalar and $\Lambda$ some constant function. All together we finally derive

$$
R_{\mu v}-\frac{R}{2} g_{\mu v}+\Lambda g_{\mu v}=\tilde{\alpha} T_{\mu v}
$$

These are Einstein's equations with a cosmological constant $\Lambda$.

\subsection{Electrostatics}

It is interesting to see what happens in the similar situation of another inverse square law, namely when we consider the electrostatic (Coulomb) force between a test particle of mass $m$ and charge $q$ at a distance $R$ from a central charge $Q$. The equivalent to the to the gravitational constant $G$ is the electric field (Coulomb) constant $k_{C}=\frac{1}{4 \pi \varepsilon}$. The acceleration of the test particle is then $a_{R}=k_{C} \frac{q Q}{m R^{2}}$. We note already here that, different to the case of gravity, where the mass $m$ drops out of the equations and hence Equation (23) is universally valid even for massless particles, the mechanism in electrostatics will be test particle-dependent. In analogy of Equation (10) we get

$$
\frac{2 k}{h} T_{a_{R}} \cdot \frac{m c R^{2}}{k_{C} q Q}=2 k T_{a_{R}} \cdot \frac{m c}{h} \cdot \frac{A_{R}}{4 \pi} \cdot \frac{1}{k_{C} q Q}=\frac{1}{\pi^{2}} .
$$

The quantity $\lambda_{C}=\frac{\hbar}{m c}$ is the (reduced) Compton wave-length and after some further calculation we get

$$
k T_{a_{R}} \cdot \frac{m c}{\hbar} \cdot \frac{A_{R}}{4}=k T_{a_{R}} \cdot \frac{A_{R}}{4 \lambda_{C}}=k_{C} q Q .
$$

Multiplying both sides with $\frac{1}{\lambda_{C}}$ we finally arrive at

$$
k T_{a_{R}} \cdot \frac{A_{R}}{4 \lambda_{C}^{2}}=\frac{k_{C} q Q}{\lambda_{C}}=E .
$$

This is the analogue to Equation (11). By using again the Unruh relation for temperature $T_{a_{R}}=\frac{\hbar}{2 \pi c} \cdot a_{R}$, we get

$$
a_{R} \cdot A_{R}=\frac{8 \pi \lambda_{C}^{2} C}{\hbar} \cdot E .
$$

This equation is the analogue to Equation (13) with $E=E(q, m, Q)$, wherein (13) we had $E=E(M)$. Assuming now that in the same manner the equilibrium is maintained during a flow of charge $\delta Q$, and hence induced energy $\delta E$, through the (local) horizon, we arrive at the equivalent to Equation (15)

${ }^{7}$ Since we have tacitly assumed in the derivation, that the groundenergy state of our systems satisfies $E_{0}=0$, it is well possible, as indicated in [2], that the constant of integration, $\Lambda$, indeed stands for this vacuum energy in case it is non-zero. 


$$
a_{R} \cdot \delta A_{R}=\frac{8 \pi \lambda_{C}^{2} c}{\hbar} \cdot \delta E=\tilde{\beta} \cdot \delta E .
$$

Due to the fact that the formulation of electrodynamics happens also in Minkowski-space, we can follow the argument in paragraph 2.1. Note that a dynamic change of charge $\delta Q$ also induces a magnetic field. To calculate the flux through the horizon we will need the energy momentum tensor $T_{\mu \nu}=F_{\mu}^{\alpha} F_{\alpha}^{v}-\frac{1}{4} g_{\mu \nu} F^{\beta \gamma} F_{\beta \gamma}$, built from the Maxwell tensor $F_{\mu \nu}=\partial_{\mu} A_{\nu}-\partial_{\nu} A_{\mu}$. The two factors $\tilde{\alpha}=\frac{8 \pi l_{P}^{2} C}{\hbar}$ and $\tilde{\beta}=\frac{8 \pi \lambda_{C}^{2} c}{\hbar}$ are very similar and use two fundamental length units, the Planck-length $l_{P}$ and the Compton length $\lambda_{C}$. They coincide, i.e. $\tilde{\alpha}=\tilde{\beta}$, if the test particle has a Planck mass $m_{P}=\sqrt{\frac{\hbar c}{G}}$.

\section{Conclusions}

In the last couple of years, we have seen a number of different ways to derive Einstein's equation by means of the holographic principle and thermodynamic equilibrium assumptions [1] [14]. A flux of energy through a (local) causal horizon causes some kinds of gravitational lensing effect in order to maintain the thermodynamic equilibrium. It seems that the other direction of reasoning, i.e. to derive the holographic principle from Einstein's equations, is much harder and the principle lies deeply hidden in the structure. Another approach starts from an information-theoretic basis, counts the maximal number of flips in a space-time volume and then carves out a corresponding number of area-elements from a horizon surface to cause it to curve [2]. The two approaches seem little related at first.

In this paper we combine the two perspectives insofar, as we follow the thermodynamic arguments to derive Einstein's equations from the holographic principle but, instead of assuming it to start with, we derive it by an information theoretic approach. We define a consistency relation across different observers, which observe different systems in thermal equilibrium, by demanding that they all agree on the same maximal number of orthogonal states (flips), which their system can pass in thermal evolution per unit of (proper) time. We apply this definition to observers in a Rindler-frame and if acceleration happens because of a gravitational or an electrostatic force, then the observers identify the available information or entropy with a quotient of the horizon area and some fundamental area units, the Planck or Compton spheres.

In fact expression (10) is very instructive and can be interpreted in the following way: if a falling observer feels an acceleration $g_{R}$ while observing a hypothetical one bit $\left(S_{\varrho}=1\right)$ system, then another observer with acceleration $g_{1}$ attributes to his system an entropy proportionate to $A_{R}$. The only system present in this set-up is space-time itself and (10) and its consequences add to the evidence that entropy is actually attributable to space-time and has an elementary unit of $l_{P}^{2}$.

\section{References}

[1] Jacobson, T. (1995) Physical Review Letters, 75, 1260-1263. http://dx.doi.org/10.1103/PhysRevLett.75.1260

[2] Lloyd, S. (2012) arXiv:1206.6559 [gr-qc]

[3] Connes, A. and Rovelli, C. (1994) Classical and Quantum Gravity, 11, 2899-2917. http://dx.doi.org/10.1088/0264-9381/11/12/007

[4] Martinetti, P. and Rovelli, C. (2003) Classical and Quantum Gravity, 20, 4919-4931. http://dx.doi.org/10.1088/0264-9381/20/22/015

[5] Smerlak, M. and Rovelli, C. (2011) Classical and Quantum Gravity, 28, Article ID: 075007. http://dx.doi.org/10.1088/0264-9381/28/17/178001

[6] Haggard, H. and Rovelli, C. (2013) Physical Review Letters, 87, Article ID: 084001.

[7] Schlatter, A. (2015) Physics Essays, 28, 296-299. http://dx.doi.org/10.4006/0836-1398-28.3.296

[8] Margolus, N. and Levitin, L. (1998) Physica D, 120, 188-195. http://dx.doi.org/10.4006/0836-1398-28.3.296

[9] Menicucci, N., Olson, S. and Milburn, G. (2014) arXiv:1108.0883 [gr-qc] 
[10] Tolman, R.C. and Ehrenfest, P. (1930) Physical Review, 36, 1791-1798. http://dx.doi.org/10.1103/PhysRev.36.1791

[11] Jacobson, T. and Parenti, P. (2003) Foundations of Physics, 33, 323-348. http://dx.doi.org/10.1023/A:1023785123428

[12] Unruh, W.G. (1976) Physical Review D, 14, 870-892. http://dx.doi.org/10.1103/PhysRevD.14.870

[13] Raychaudhuri, A.K. (1955) Physical Review, 98, 1123-1126. http://dx.doi.org/10.1103/PhysRev.98.1123

[14] Verlinde, E. (2011) Journal of High Energy Physics. arXiv:1001.0785 [hep-th] 